\title{
Efficient delivery of HBV NLS siRNAs into HepG2.2.15 cells for HBV inhibition through novel recombinant preS1-tP proteins
}

\author{
YANLI ZENG $^{1 *}$, ZIXI LI $^{2 *}$, JIA SHANG $^{1}$ and YI KANG ${ }^{1}$ \\ ${ }^{1}$ Department of Infectious Diseases, Zhengzhou University People's Hospital, Henan University People's Hospital, Henan \\ Provincial People's Hospital, Zhengzhou, Henan 450003; ${ }^{2}$ Tongji Medical College of Huazhong University of Science \\ and Technology Affiliated Hospital of Traditional Chinese and Western Medicine, Wuhan, Hubei 430030, P.R. China
}

Received January 15, 2018; Accepted May 8, 2018

DOI: $10.3892 /$ ijmm.2018.3681

\begin{abstract}
Hepatitis B virus (HBV) infection and related liver complications remain severe public health problems worldwide. Previous investigations have shown that small interfering (si)RNAs can offer an effective strategy for the treatment of chronic hepatitis B. The present study aimed to develop a novel siRNA-delivering system of therapeutic HBV nuclear localization sequence (NLS) siRNAs using the recombinant preS1-truncated protamine (tP) proteins. The preS1 region of the LHB was used in place of $\mathrm{scFv}$ to construct the recombinant preS1-tP proteins, which were applied to deliver siRNAs targeting the HBV NLS to inhibit HBV replication and infection in HepG2.2.15 cells overexpressing sodium taurocholate cotransporting polypeptide (NTCP). The results revealed that HepG2.2.15 cells with stable NTCP expression (HepG2.2.15-NTCP cells) transfected with the recombinant lentivirus showed increased expression of NTCP genes. The HBV NLS siRNAs significantly suppressed HBV mRNA content and levels of HBsAg and HBeAg in the HepG2.2.15-NTCP cells. Recombinant preS1-tP proteins tagged with His and glutathione S-transferase were found to enter into HepG2.2.15-NTCP cells and bind with DNA. The HBV NLS siRNAs were delivered into HepG2.2.15-NTCP cells by recombinant preS1-tP proteins, which resulted in decreased expression of HBV mRNA, HBsAg and HBeAg, HBV DNA and covalently closed circular DNA in the HepG2.2.15-NTCP cells. Therefore, the recombinant preS1-tP
\end{abstract}

Correspondence to: Dr Yi Kang, Department of Infectious Diseases, Zhengzhou University People's Hospital, Henan University People's Hospital, Henan Provincial People's Hospital, 7 Weiwu Avenue, Jinshui, Zhengzhou, Henan 450003, P.R. China

E-mail: zyldd1212@126.com

*Contributed equally

Key words: hepatitis B virus, sodium taurocholate cotransporting polypeptide, nuclear localization sequence small interfering RNAs, HepG2.2.15 cell, recombinant preS1-truncated protamine proteins proteins successfully delivered NLS siRNAs into HepG2.2.15 cells and repressed HBV infection and replication.

\section{Introduction}

Infection with hepatitis B virus (HBV), which usually causes chronic hepatitis $\mathrm{B}$ (CHB), is a cosmopolitan public health problem which is highly endemic and associated with a high mortality rate and heavy societal burden $(1,2)$. A mortality rate of $>1,000 /$ year has been attributed to liver failure, liver cirrhosis and hepatocellular carcinoma caused by long-term HBV infection, which accounts for $>15 \%$ of those succumbing to chronic diseases globally $(2,3)$. Although the prevalence of HBV in certain highly endemic regions, including rural China, has shown improvement, the large absolute number of $\mathrm{HBV}$-infected susceptible individuals in the population remains a potential threat (4). Interferon and nucleoside acid analogs are the major therapeutic agents that have been used for the treatment of CHB by delaying disease progression, however, these are limited by severe drug resistance and relapse following drug withdrawal (2).

The covalently closed circular DNA (cccDNA), which is formed in the liver cell nucleus following the binding of HBV with cellular receptors during HBV infection, functions as the main template for the replication of HBV particles and has been accepted as the main reason for the difficulty in HBV eradication $(5,6)$. Due to the persistence of viral cccDNA, which is transcriptionally active in the liver cell nucleus, lifelong therapy is required using antiviral drugs, which are currently unable to directly target the formation of cccDNA $(7,8)$. The nuclear localization sequence (NLS) of HBV core protein can be recognized by and is associated with importin, thus having an important function in transferring HBV relaxed-circular serum DNA into the hepatocyte nucleus for cccDNA formation (9). Small interfering (si)RNAs targeting HBV NLS can induce the degradation of mRNAs responsible for HBV core protein nuclear entry, and lead to the subsequent inhibition of cccDNA formation and HBV replication (10), demonstrating the potential of HBV NLS siRNAs in the treatment of HBV infection. However, the effective inhibition of cccDNA formation by HBV NLS siRNAs depends on the specific delivery of interfering molecules into the infected hepatocytes. 
The entry of HBV into hepatocytes follows multiple steps, including the initial attachment of HBV to host cell surface proteoglycans, early entry following high-affinity binding by specific receptors, endocytosis-mediated internalization, and fusion with the cellular membrane (11). Three HBV surface proteins are important for the entry processes, including the large HBV surface proteins (LHBs) which contain the preS1, preS2 and $\mathrm{S}$ regions, the middle surface proteins containing the preS2 and $\mathrm{S}$ regions, and the small surface proteins that comprise only the S regions (11). Among these three characteristic regions in HBV surface proteins, the $\mathrm{S}$ and preS1 regions are reported to be important in HBV infection $(11,12)$. The sodium taurocholate cotransporting polypeptide (NTCP) has been identified as the cellular entry receptor for HBV (13), which has attracted increased attention regarding the molecular mechanisms underlying the entry steps of HBV infection. NTCP, also known as solute carrier family 10A1 (14), was previously demonstrated to be a transporter in the hepatocyte basolateral membrane and function in conjugated bile salt uptake by hepatic cells (11). Investigations have shown that NTCP can function as an HBV entry receptor by directly binding preS1-derived lipopeptide, which was supported by the close correlation between the expression of NTCP and HBV susceptibility in multiple cells, and the significantly enhanced HBV susceptibility conferred by the ectopic expression of NTCP in HepG2 cells $(11,13,15,16)$. These findings suggest that hepatocytes modified to overexpress NTCP may be useful cellular models for the development and screening of novel anti-HBV targeting therapy. The specific interaction between preS1 and NTCP may be examined for the specific delivery of therapeutic agents into infected hepatocytes for targeting treatment.

Based on the roles of HBV surface proteins and cellular receptors in viral infection and disease progression, the specific delivery of siRNAs by the single chain variable fragment (scFv), which specifically recognizes HBV surface antigen (HBsAg), the specific antigen expressed on hepatocytes infected by HBV, has been shown as a promising approach for inhibiting HBV gene expression $(17,18)$. The fusion protein containing $\mathrm{scFv}$ and truncated protamine (tP), which binds with DNA with high affinity (19), can be internalized by HepG2.2.15 cells and effectively inhibit HBV gene expression by the siRNAs delivered by the recombinant proteins $(17,18)$. However, the efficiency of such a delivery system may be limited by the neutralizing effect of abundantly existing HBsAg in the serum of patients with CHB. Therefore, in the present study, the preS1 region of the LHB was used in place of $\mathrm{scFv}$ to construct the recombinant preS1-tP proteins, which were applied to deliver siRNAs targeting the HBV NLS to inhibit HBV replication and infection in HepG2.2.15 cells overexpressing NTCP.

\section{Materials and methods}

Cell lines and culture. The HepG2.2.15 cells (cat.no. YB-ATCC-2242) and 293T cells (cat. no. CRL-3216 ${ }^{\mathrm{TM}}$ ) were purchased from the Human American Type Culture Collection (Manassas, VA, USA), and cultured in DMEM (Gibco; Thermo Fisher Scientific, Inc., Waltham, MA, USA) supplemented with $10 \%$ fetal bovine serum (FBS)
(Sigma-Aldrich; Merck KGaA, Darmstadt, Germany), $2 \mathrm{mM}$ L-glutamine, $100 \mathrm{mg} / \mathrm{ml}$ streptomycin and $100 \mathrm{mg} / \mathrm{ml}$ penicillin at $37^{\circ} \mathrm{C}$ in $5 \% \mathrm{CO}_{2}$.

Generation of HepG2.2.15 cells stably expressing the NTCP gene. To generate HepG2.2.15 cells stably expressing the NTCP gene, the human NTCP coding DNA sequences were cloned by polymerase chain reaction (PCR) using the following specific primers: NTCP, forward 5'-ATGCTCTAGAGCCAC CATGGAGGCCCAC-3' and reverse 5'-ATGGGGATCCTC AGGCTGTGCAAGGGGAG-3'. The PCR products were then digested using two restriction endonucleases EcoRI and $X b a \mathrm{I}$, and then ligated to the LV003 plasmid (Promega Corporation, Madison, WI, USA) for the construction of recombinant LV003-NTCP plasmids. Establishment of the HepG2.2.15 cells stably expressing the NTCP gene was performed as previously described with minor modifications (20). Briefly, the packaging procedure was performed by the co-infection of 293T cells with pGag/Pol $(5 \mu \mathrm{g})$, pRev $(5 \mu \mathrm{g})$ and pVSV-G $(5 \mu \mathrm{g})$ supplied by Shanghai GenePharma Co., Ltd. (Shanghai, China) following the protocol described by the manufacturer. The cell culture medium containing the recombinant virus was collected and transfected into HepG2.2.15 cells using lipofectamine 2000 (Invitrogen; Thermo Fisher Scientific, Inc.), according to the manufacturer's instructions. At 3 days post-infection, the HepG2.2.15 cells showing stable NTCP gene expression were screened using DMEM containing puromycin, as previously described (21). The expression of NTCP in HepG2.2.15 cells was finally confirmed using fluorescence microscopy and reverse transcription-quantitative (RT-q)PCR analysis 10 days following the screening.

$R T$ - $q P C R$ analysis. The relative mRNA levels of NTCP and associated genes were analyzed by RT-qPCR analysis in the present study. The cells were washed with PBS three times, and total RNA samples from the cultured cells were extracted using TRIzol reagent (cat. no. 15596018) in strict accordance with the manufacturer's protocol (Thermo Fisher Scientific, Inc.). The cDNA samples were then synthesized using random primers and the Prime Script RT Master Mix kit (cat. no. RR036a; Takara Bio, Inc., Otsu, Japan) according to the manufacturer's protocol. PCR was carried out in a $20 \mu \mathrm{l}$ reaction mixture containing $7.5 \mu 1 \mathrm{GoTaq}^{\circledR}$ Green Master Mix (Promega Corporation), $1.5 \mu \mathrm{l}$ of cDNA, $1 \mu \mathrm{l}$ of each primer, $9.0 \mu 1$ nuclease-free water with the following conditions, $95^{\circ} \mathrm{C}$ for $120 \mathrm{sec}$, followed by 40 cycles at $60^{\circ} \mathrm{C}$ for $30 \mathrm{sec}$. The primer sequences were as follows, NTCP: Forward, 5'-AGG GAGGAGGTGGCAATCAAGAGTGG-3', reverse, 5'-CCG GCTGAAGAACATTGAGGCACTGG-3'; HBV: Forward, 5'-CCGTCTGTGCCTTCTCATCTGC-3', reverse, 5'-ACC AATTTATGCCTACAGCCTC-3'; GAPDH: Forward, 5'-GAG TCAACGGATTTGGTCGT-3', reverse, 5'-CCCAGTAGC AGTTCAGGTGG-3'. The gene expression level was finally determined by RT-qPCR analysis using the SYBR Select Master Mix kit (cat. no. 4472908; Thermo Fisher Scientific, Inc.) following the manufacturer's protocol. GAPDH was used as the internal control for gene expression level quantitation. At least three biological repeats and three technical replicates were performed for the statistical comparison of gene expression. Data were quantified using the $\Delta \Delta^{\mathrm{Cq}}$ method normalizing to 
Table I. siRNA sequences for sodium taurocholate cotransporting polypeptide knockdown.

$\begin{array}{lll}\text { SiRNA } & \text { Sense (5'-3') Antisense (5'-3') }\end{array}$

$\begin{array}{ll}\text { siRNA-1 } & \text { AAGAUCUCAAUCUCGGGAAUC } \\ \text { siRNA-2 } & \text { CAGGUCCCCUAGAAGAAGAAC } \\ \text { siRNA-3 } & \text { AACACUUCCGGAAACUACUGU }\end{array}$

siRNA, small interfering RNA.

GAPDH as reference genes, and further normalized to the lowest-expressing sample for comparison (22).

Suppression of $H B V$ by NLS siRNA. The knockdown of the gene expression of NTCP in HepG2.2.15 cells stably expressing the NTCP gene was performed using Lipofectamine ${ }^{\mathrm{TM}}$ RNAiMAX reagent supplied Invitrogen; Thermo Fisher Scientific, Inc. (cat. no. 13778-075) following the manufacturer's protocol. RNAi duplex $(\sim 6$ pmol) was diluted in $100 \mu \mathrm{l}$ DMEM without serum and gently mixed, which was added to $1 \mu$ l Lipofectamine ${ }^{\mathrm{TM}}$ RNAiMAX, mixed gently, and then incubated at room temperature for $15 \mathrm{~min}$. The HepG2.2.15 cells stably expressing NTCP were cultured in 24-well plates at $\sim 70 \%$ confluence, and then diluted with DMEM to $1 \times 10^{5}$ cells $/ \mathrm{ml}$. The cells $(\sim 0.5 \mathrm{ml})$ were then mixed with the previously prepared RNAi duplex and Lipofectamine ${ }^{\mathrm{TM}}$ RNAiMAX mixture, mixed gently and incubated for $72 \mathrm{~h}$ at $37^{\circ} \mathrm{C}$ with $5 \% \mathrm{CO}_{2}$. The knockdown of the gene expression of NTCP was confirmed by RT-qPCR, followed by the analysis of HBsAg and HBeAg antigens through an enzyme-linked immunosorbent assay (ELISA). For the successful knockdown of gene expression, at least three biological and technical replicates were performed. The siRNA sequences used for suppressing the gene expression if NTCP are listed in Table I.

ELISA. The expression levels of HBsAg (cat. no. KA0286) and $\mathrm{HBeAg}$ (cat. no. KA0290) antigens in the HepG2.2.15 cells stably expressing NTCP were determined by the ELISA method using the commercially available kits supplied by Novus Biologicals, Ltd. (Cambridge, UK) following the manufacturer's protocol. The quantification of antigen levels was performed by measuring the absorbance at $405 \mathrm{~nm}$ using a microtiter plate spectrophotometer. At least three biological and technical replicates were performed for accurate quantification by statistical analysis.

Expression and purification of recombinant preS1-tP protein in Escherichia coli. The recombinant preS1-tP proteins were expressed and purified in BL-21 strains of E. coli (American Type Culture Collection, Manassas, VA, USA) using regular protein expression and purification protocols. Briefly, the preS1 region of the HBV surface protein was cloned using the PCR method. The PCR products of preS1 and tp DNA fragments were ligated into the p-GEX-6P-1 plasmids, which were used for the transformation of $E$. coli BL21 strains Monoclones with the recombinant plasmid were then screened by culture in LB medium containing $34 \mu \mathrm{g} / \mathrm{ml}$ chloromycetin and $50 \mu \mathrm{g} / \mathrm{ml}$ ampicillin. The large-scale expression and purification of recombinant proteins were performed by overnight induction with $0.5 \mathrm{mM}$ isopropyl $\beta$-D-thiogalactoside (IPTG) at $30^{\circ} \mathrm{C}$ with agitation. The $E$. coli cells were collected by centrifugation at $8 \times \mathrm{g}$ over night at $37^{\circ} \mathrm{C}$ and lysed by sonication. The supernatant was then purified by two rounds of affinity chromatography, and visualized using $12 \%$ SDS-PAGE electrophoresis.

Immunofluorescence. As a final examination of the functioning of recombinant preS1-tP proteins in delivering HBV NLS siRNAs, the NLS siRNA fragment was synthesized and labeled with $\mathrm{Cy} 3$ fluorescent (Cy3 siNLS), with a shNLS plasmid expressing red fluorescence protein also applied. Subsequently, $5 \mu \mathrm{g}$ recombinant preS1-tP proteins were mixed with 150 pmol Cy3 siNLS or $2 \mu \mathrm{g}$ shNLS in $0.2 \mathrm{M}$ $\mathrm{NaCl}$ for $30 \mathrm{~min}$, and incubated with the HepG2.2.15 cells for $48 \mathrm{~h}$. The entry of recombinant protein with glutathione S-transferase (GST) and His tags into hepatocytes was first determined by immunofluorescence using anti-His antibodies (cat. no. ab1187; Abcam, Cambridge, UK). The HepG2.2.15 cells stably expressing NTCP were grown on plates, and covered with 2-3 mm 4\% formaldehyde prepared with PBS solution for $15 \mathrm{~min}$ at room temperature. Following washing for 5 min with PBS solution three times, the cell plates were then blocked with 5\% lipid-free milk solution for $1 \mathrm{~h}$ at room temperature, incubated with anti-His antibodies (diluted at 1:1,000) overnight $4^{\circ} \mathrm{C}$, rinsed three times with PBS for $5 \mathrm{~min}$ each, incubated with fluorochrome-conjugated secondary antibody (1:2,000; cat. no. ab205718; Abcam) for $2 \mathrm{~h}$ in the dark at room temperature, and rinsed again three times with PBS for 5 min each. Prolong ${ }^{\circledR}$ Gold Antifade reagent with DAPI was then added (cat. no. 8961; Cell Signaling Technology, Inc., Danvers, MA, USA) overnight at room temperature. The existence of recombinant proteins in hepatocytes were finally determined using fluorescence microscopy.

Western blot analysis. The HepG2.2.15 cells stably expressing NTCP were washed three times with PBS, collected by centrifugation at $150 \mathrm{xg}$ for $5 \mathrm{~min}$ at $37^{\circ} \mathrm{C}$, and then used for total protein extraction. Protein concentrations were determined using the Bradford method. The cells were lysed by incubating with RIPA buffer containing 1X Halt Protease Inhibitor Cocktail (Thermo Fisher Scientific, Inc.). Following boiling for $5 \mathrm{~min}$ at $100^{\circ} \mathrm{C}$ in sample buffer $(300 \mathrm{mM}$ Tris- $\mathrm{HCl}$, $1 \%$ SDS, 3.5 M $\beta$-mercaptoethanol, 5 mM PMSF and $6 \%$ glycerol and $0.05 \%$ bromophenol blue), the protein samples $(40 \mu \mathrm{g})$ 
were separated by $10 \%$ SDS-PAGE, transferred onto PVDF membranes, blocked with 5\% lipid-free milk, and incubated with anti-GST antibodies (1:1,000; cat. no. ab19256; Abcam) and anti-GAPDH (1:2,000; ab9485; Abcam) overnight at $4^{\circ} \mathrm{C}$ and secondary antibodies (1:2,000; cat. no. ab205718; Abcam) for $2 \mathrm{~h}$ at room temperature in TBST buffer successively. The protein levels were finally visualized by adding enhanced chemiluminescence solution (Amersham; GE Healthcare Life Sciences, Chalfont, UK).

Statistical analysis. The SPSS 18.0 software package (SPSS, Inc., Chicago, IL, USA) was used for statistical analysis in the present study. The significance of differences between groups was statistically analyzed using Student's t-test. At least three biological repeats were used for statistical evaluation. $\mathrm{P}<0.05$ was considered to indicate a statistically significant difference.

\section{Results}

Establishment of HepG2.2.15 cells overexpressing NTCP. Due to the importance of NTCP protein in the infection and replication of HBV, hepatocytes overexpressing the NTCP gene may be applied as an ideal cellular model for the development and screening of novel anti-HBV therapies. The coding DNA sequences of the human NTCP gene were amplified by PCR, and ligated onto the LV003 plasmid. The recombinant LV003-NTCP plasmid was then used to co-infect 293 cells with $\mathrm{pGag} / \mathrm{Pol}, \mathrm{pRev}$ and pVSV-G plasmids for packaging into the lentivirus. Finally, the virus collected from the cell culture medium was used to infect HepG2.2.15 cells for the screening of cells stably expressing the NTCP gene (Fig. 1A). For further confirmation, the gene expression level of NTCP in HepG2.2.15 cells was verified using the RT-qPCR method, which also showed markedly elevated gene expression of NTCP in the HepG2.2.15 cells infected with the virus (Fig. 1B). These results showed the successful establishment of HepG2.2.15 cells overexpressing the human NTCP gene, which were used for the development of a novel siRNA therapy for HBV in the following experiments.

Suppression of HBV replication and infection in HepG2.2.15 cells using HBV NLS siRNAs. Previous studies have shown that HBV NLS siRNAs can effectively suppress HBV infection and replication in hepatocytes (17). For the development of a novel HBV NLS siRNA delivery system using recombinant preS1-tP proteins, three pairs of HBV NLS siRNAs were designed, as described above. Subsequent to being transfected with these HBV NLS siRNAs, the total RNA samples of HepG2.2.15 cells stably expressing the NTCP gene were extracted and used for quantitative analysis of the mRNA expression of HBV. The results showed that the mRNA levels of HBV in the HepG2.2.15 cells stabling expressing HBV mRNA were markedly suppressed by the transfection with all three of the HBV NLS siRNAs (Fig. 2A). To confirm the replication of HBV in these HepG2.2.15 cells, the expression levels of two HBsAgs ( $\mathrm{HBeAg}$ and $\mathrm{HBeAg}$ ), were measured using ELISA methods. The ELISA results also demonstrated that NLS siRNA-2 and NLS siRNA-3 significantly inhibited the levels of HBsAg in the HepG2.2.15 cells, and siRNA-3 significantly downregulated the level of HBeAg in the HepG2.2.15 cells (Fig. 2B and C). Among the three HBV siRNAs used in this screening, siRNA-3 exerted the most marked inhibitory effect on the activity of HBV in the HepG2.2.15 cells, and was used for the subsequent experiments.

Purification of recombinant preS1-tP proteins. For the successful delivery of HBV NLS siRNAs into hepatocytes for the suppression of HBV infection and replication, the preS1 regions of the LHB gene and tp fragment were cloned separately, and then ligated to the p-GEX-6P-1 plasmids. The recombinant preS1-tP proteins tagged with GST were expressed in and purified from the E. coli BL21 strain. The results of SDS-PAGE showed that the recombinant preS1-tP proteins were expressed in E. coli BL21 cells by induction with IPTG at $37^{\circ} \mathrm{C}$, but not at $20^{\circ} \mathrm{C}$ (Fig. $3 \mathrm{~A}$ and B). The significant protein band between the 35 and $45 \mathrm{kDa}$ protein markers indicated the GST-tagged recombinant preS1-tP protein (Fig. 3A and B). The recombinant preS1-tP proteins were then expressed and purified on a large scale by affinity chromatography using glutathione-agarose and Ni sepharose (Fig. 3C), which was then applied for the construction of a novel delivery system of HBV NSL siRNAs.

Recombinant preS1-tP protein entry into HepG2.2.15 cells and DNA binding. For successful establishment of a novel delivery system of HBV NLS siRNAs, the entry of the carriers, the recombinant preS1-tP proteins in the present study, into the target cells and their high-affinity binding with host DNA are key steps. Therefore, the slides of HepG2.2.15 cells stably expressing the human NTCP gene were incubated with purified recombinant preS1-tP proteins overnight. The immunofluorescence assay using anti-His antibodies showed that the recombinant preS1-tP proteins had successfully entered into the HepG2.2.15 cells stably expressing NTCP (Fig. 4A). For further verification of the recombinant protein entry into the HepG2.2.15 cells, total proteins of the cells incubated with the recombinant preS1-tP proteins overnight were extracted and subjected to an immunoblotting assay using anti-GST antibodies. The results of the western blot analysis also demonstrated entry of the recombinant proteins into the HepG2.2.15 cells (Fig. 4B and C). However, the results of the immunofluorescence assay showed that the recombinant proteins were mainly distributed in the cytoplasm of the HepG2.2.15 cells. As a preliminary assessment of the capacity of recombinant preS1-tP proteins to bind host cell DNA, $1 \mu \mathrm{g}$ short hairpin (sh) NLS plasmids were mixed with 0,250 or $500 \mathrm{ng}$ recombinant preS1-tP proteins, and then analyzed by electrophoresis. This also revealed the binding of recombinant preS1-tP proteins with shNLS plasmids in vitro (Fig. 4D). Therefore, the results showed that the recombinant preS1-tP proteins were able to enter into the HepG2.2.15 cells by simple co-incubation and bind the DNA in the in vitro assay, demonstrating the potential of recombinant preS1-tP proteins for the delivery of NLS siRNA into HepG2.2.15 cells for treatment of HBV infection.

Inhibition of $H B V$ infection and production of cccDNA by $N L S$ siRNA delivered by recombinant preSl-tP proteins in HepG2.2.15 cells. Fluorescence microscopy showed that the Cy3 siNLS and shNLS had been successfully carried into the HepG2.2.15 cells by the recombinant preS1-tP 
A

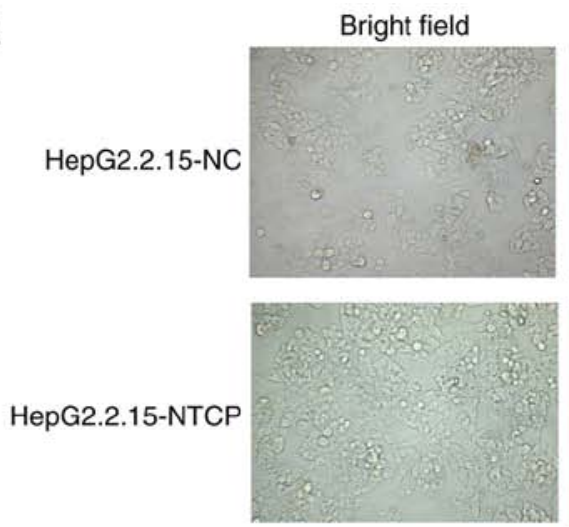

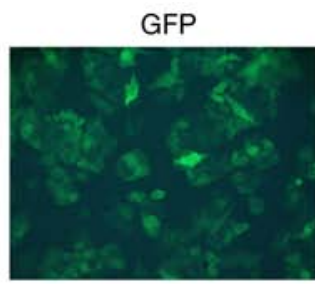

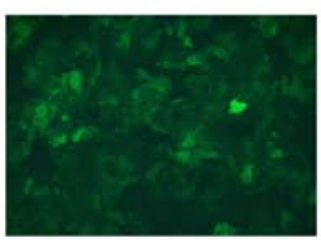

B

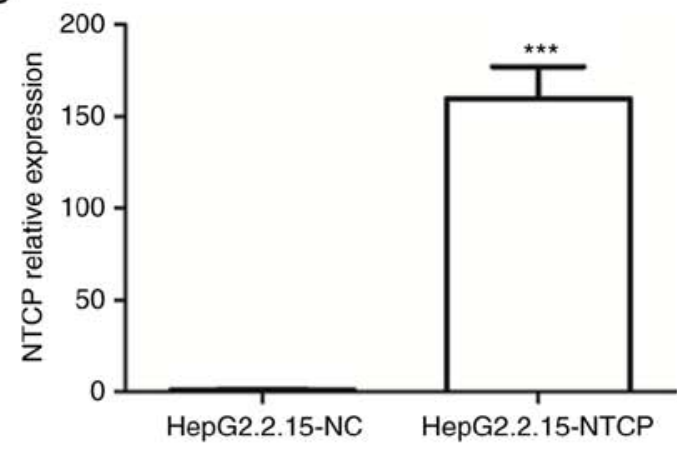

Figure 1. Overexpression of NTCP gene in HepG2.2.15 cells. (A) Analysis of the gene expression of NTCP by GFP signals using fluorescence microscopy (magnification, x200). (B) Gene expression level of NTCP in HepG2.2.15 cells by reverse transcription-quantitative polymerase chain reaction analysis.

${ }^{* * *}$ Compared with the NC group, ${ }^{* * *} \mathrm{P}<0.001$. NC, negative control; NTCP, sodium taurocholate cotransporting polypeptide.

A

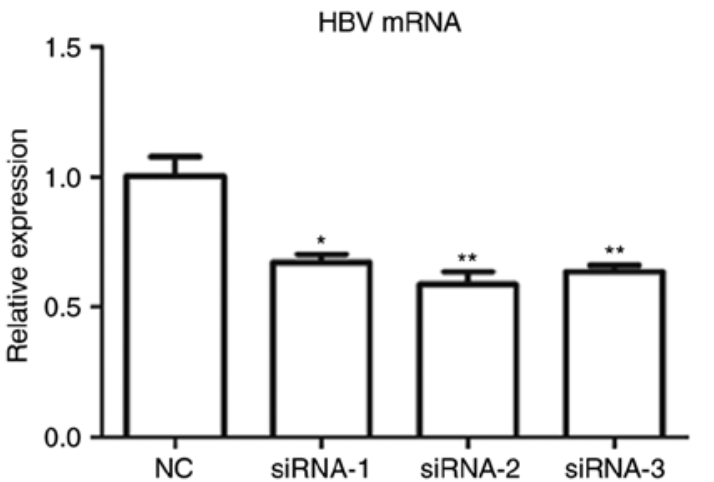

B

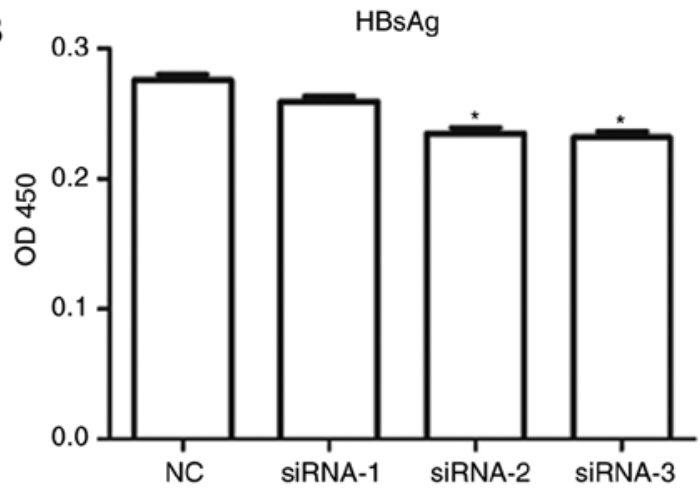

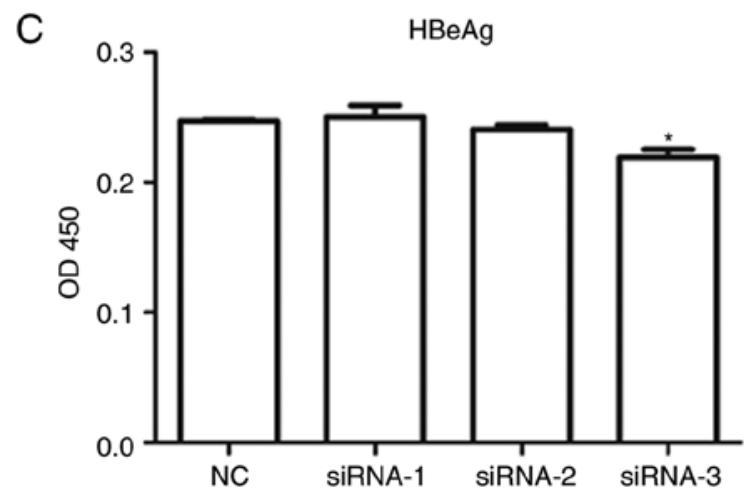

Figure 2. Suppression of HBV infection in HepG2.2.15 cells using NLS siRNAs. (A) mRNA expression of HBV in HepG2.2.15 cells stably expressing the NTCP gene by NLS siRNAs, determined using reverse transcription-quantitative polymerase chain reaction analysis. Enzyme-linked immunosorbent assay of the expression of HBV surface antigens (B) HBsAg and (C) HBeAg in HepG2.2.15 cells stably expressing the NTCP gene. ${ }^{*}$ and ${ }^{* *}$, compared with the NC group, ${ }^{*} \mathrm{P}<0.05,{ }^{* *} \mathrm{P}<0.01$. HBV, hepatitis B virus; siRNAs, small interfering RNAs; NC, negative control; NTCP, sodium taurocholate cotransporting polypeptide; NLS, nuclear localization sequence; HBsAg, Hepatitis B surface antigen; HBeAg, hepatitis B e antigen.

proteins (Fig. 5A). To show the effect of siNLS and shNLS on HBV function in HepG2.2.15 cells, the mRNA levels of HBV in HepG2.2.15 cells were determined using the RT-qPCR method. It was observed that the HepG2.2.15 cells incubated with siNLS or shNLS exhibited significantly repressed mRNA levels of HBV, compared with the cells incubated with the recombinant preS1-tP proteins alone (Fig. 5B). In addition, as demonstrated by ELISA, the levels of HBsAg and $\mathrm{HBeAg}$ were also markedly downregulated in the HepG2.2.15 cells, compared with the control (Fig. 5C and D). It was found that the HBV DNA content in HepG2.2.15 cells incubated with siNLS or shNLS was also markedly decreased, compared with that in the cells incubated with only the recombinant proteins (Fig. 5E), showing the effect of preS1-tP protein-delivered NLS siRNAs in suppressing HBV replication. Finally, the levels of HBV cccDNA in HepG2.2.15 cells showed a similar decrease in the HepG2.2.15 cells induced by incubation with preS1-tP proteins and HBV NLS siRNAS (Fig. 5F). Taken together, these results confirmed that the preS1-tP protein acted as the effective deliverer of HBV NLS siRNA for the inhibition of HBV infection and replication in hepatocytes. 


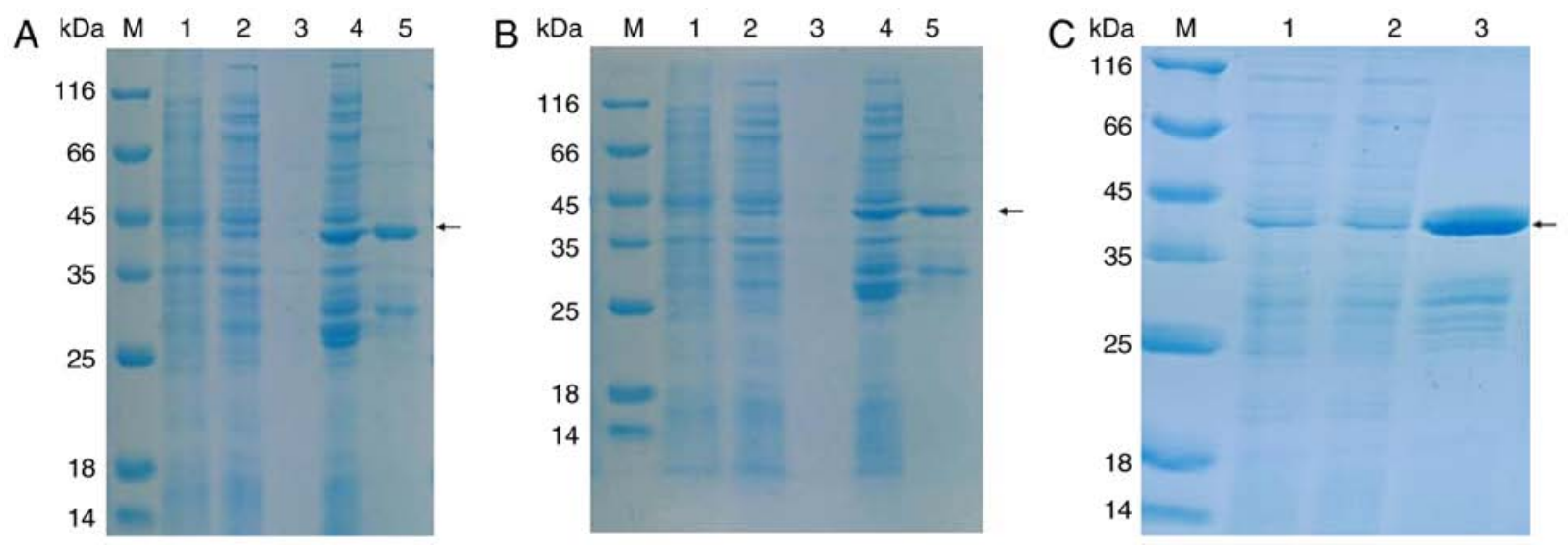

Figure 3. Expression and purification of recombinant preS1-tP proteins in E. coli. Expression of recombinant preS1-tP proteins in E. coli BL-21 cells on (A) small and (B) large scales. Lane 1 shows total protein prior to IPTG induction; lanes 2 and 3, show the supernatant and precipitate following IPTG induction at $20^{\circ} \mathrm{C}$; lanes 4 and 5 show the supernatant and precipitate following IPTG induction at $37^{\circ} \mathrm{C}$. The arrow indicates recombinant preS1-tP protein. (C) Purification of recombinant preS1-tP proteins in E. coli BL-21 cells by affinity chromatography. M, protein marker ladder; lane 1, loaded cell lysate; lane 2, flow through during affinity chromatography; lane 3, elute during affinity chromatography. Arrows indicate recombinant preS1-tP protein. E. coli, Escherichia coli, tp, truncated protamine; IPTG, isopropyl $\beta$-D-thiogalactoside.
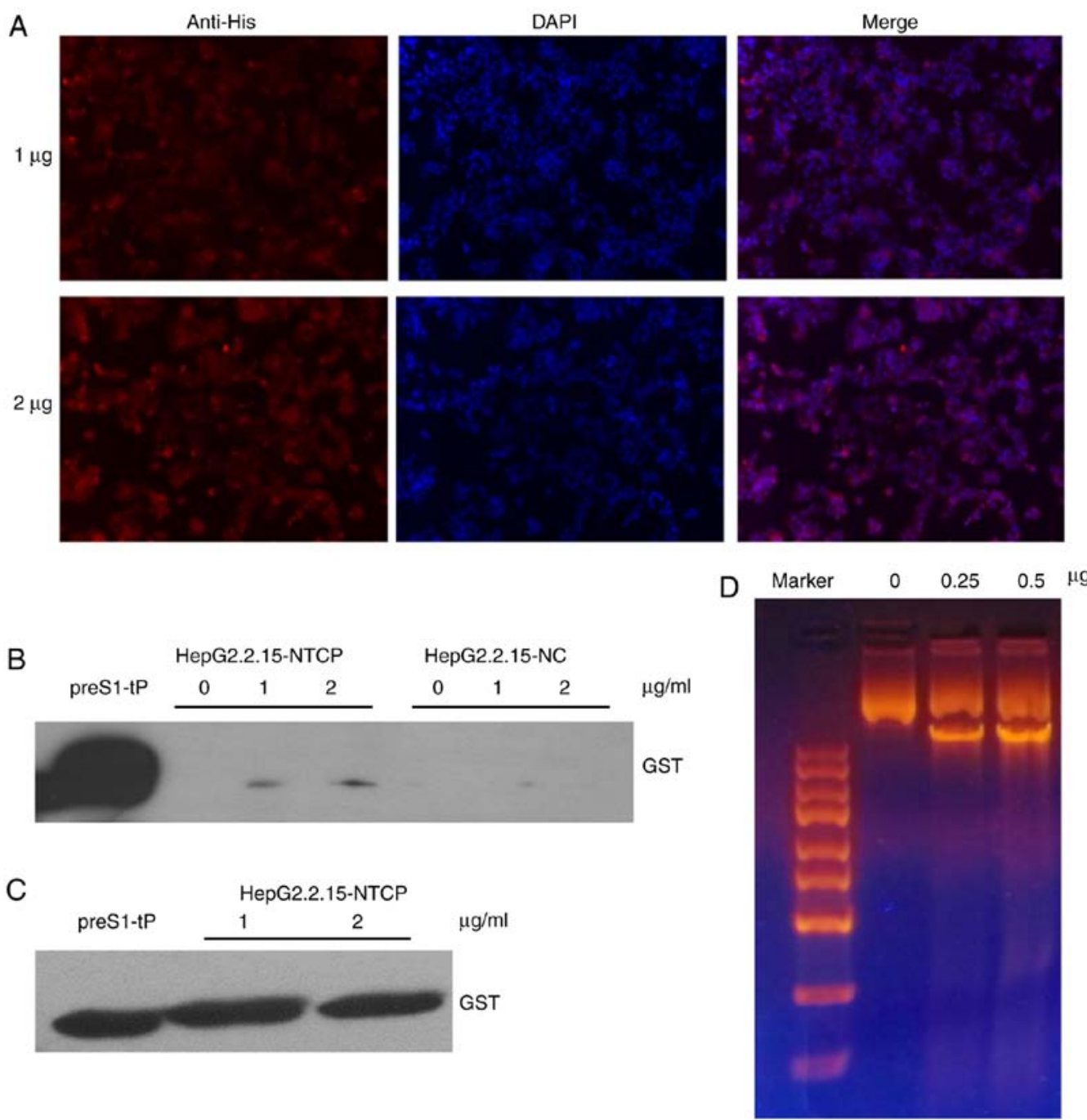

Figure 4. Entry of recombinant preS1-tP proteins into hepatocytes and its binding with DNA. (A) Existence of recombinant preS1-tP proteins in HepG2.2.15 cells by immunofluorescence (magnification, x200). HepG2.2.15 cells were pre-incubated with recombinant preS1-tP proteins (1 and $2 \mu \mathrm{g})$ overnight. DAPI staining was used to indicate the cell nucleus. (B) Detection of recombinant preS1-tP proteins in HepG2.2.15 cells pre-incubated with recombinant proteins by western blot analysis using anti-GST antibodies. (C) Verification of the recombinant preS1-tP protein entry into the HepG2.2.15 cells. (D) Electrophoresis showing separation of the complexes of recombinant preS1-tP proteins and shNLS plasmids. The recombinant preS1-tP proteins and shNLS plasmids were pre-incubated in vitro at room temperature in $0.2 \mathrm{M} \mathrm{NaCl}$ for $30 \mathrm{~min}$. DAPI, 4',6-diamidino-2-phenylindole; tP, truncated protamine; NTCP, sodium taurocholate cotransporting polypeptide; NC, negative control; GST, glutathione S-transferase. 

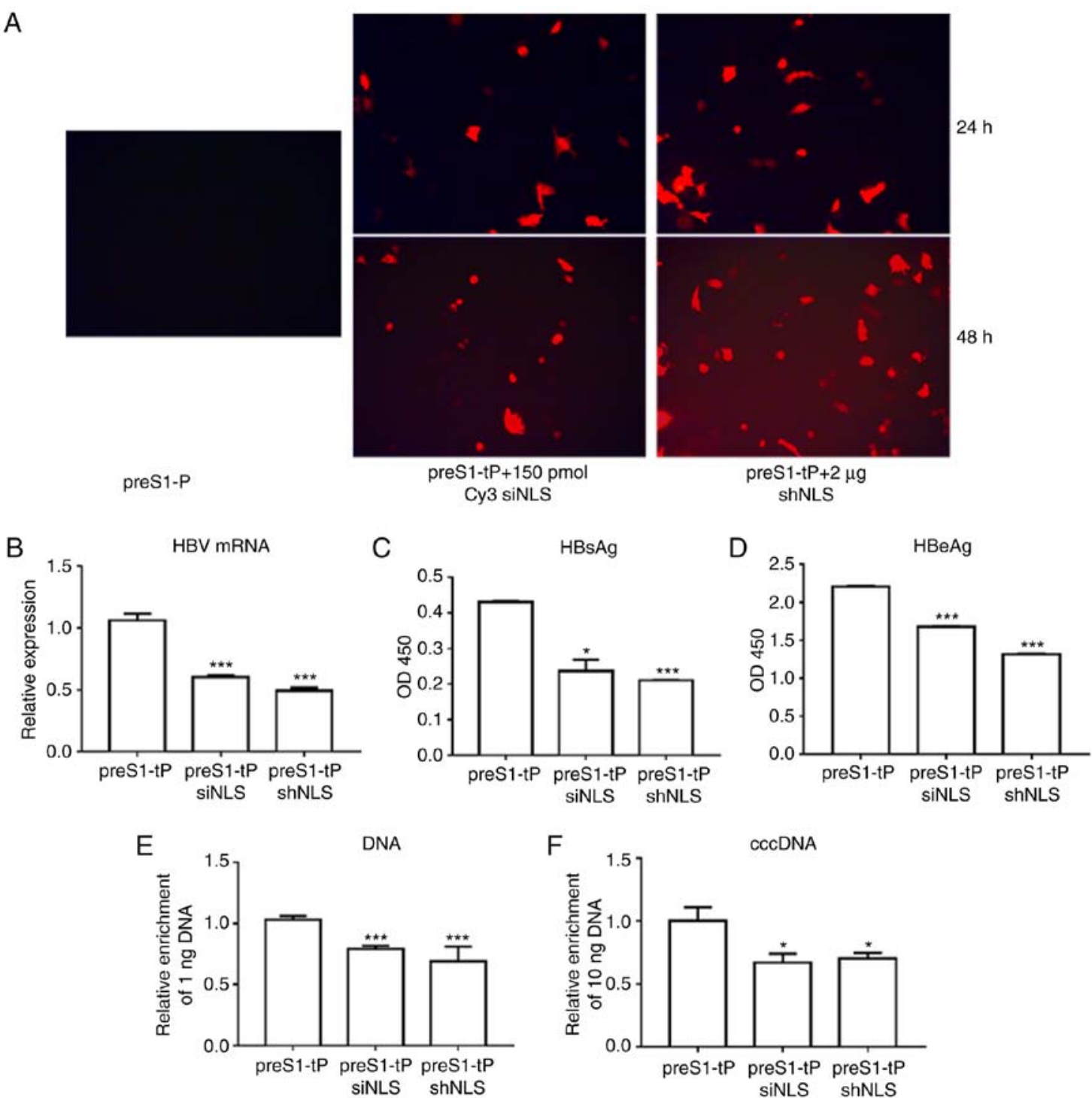

Figure 5. Inhibition of HBV infection by NLS siRNAs delivered by preS1-tP proteins. (A) Entry of HBV NLS siRNAs into HepG2.2.15 cells through recombinant preS1-tP proteins by immunofluorescence (x200). Levels of (B) HBV mRNA, (C) HBsAg, (D) HBeAg, (E) HBV DNA and (F) cccDNA in HepG2.2.15 cells co-incubated with recombinant preS1-tP proteins and NLS siRNAs. ${ }^{* * * *}$ and ${ }^{* * * * *}$, compared with the preS1-tP group, ${ }^{*} \mathrm{P}<0.05,{ }^{{ }^{* * *}} \mathrm{P}<0.01,{ }^{* * * *} \mathrm{P}<0.001$. HBV hepatitis B virus; NLS, nuclear localization sequences; cccDNA, covalently closed circular DNA; HBsAg, hepatitis B surface antigen; HBeAg, hepatitis B e antigen; siRNA, small interfering RNA; shRNA, short hairpin RNA; tP, truncated protamine.

\section{Discussion}

Although the wide application of anti-HBV vaccines has significantly decreased the incidence of HBV infection, there were still $>350,000,000$ patients with CHB globally in 2016 (23). The treatment and eradication of HBV infections have been limited by the unavailability of effective therapeutic drugs and methods to completely remove the cccDNA in the infected hepatocytes. Due to the significance of the human NTCP gene during HBV infection and entry, the establishment of hepatocytes with stable NTCP gene expression has been widely applied as a cellular model for the investigation of CHB pathology, and for the screening of therapeutic agents and methods for the treatment of HBV (11,20,24-26). Guided by the same rationale, the present study used HepG2.2.15 cells expressing the NTCP gene to develop a novel method for delivering HBV NLS siRNAs into HepG2.2.15 cells. Previous studies have shown that the delivery of siRNA molecules through $\mathrm{s}$ fusion protein containing $\mathrm{scFv}$ and $\mathrm{tP}$ may be a novel method for the inhibition of HBV infection and replication, considering the improved specificity and promoted affinity $(17,18,27,28)$. However, it is possible that the high level of existing HBsAg in patients with $\mathrm{CHB}$ may be a concern for the application of recombinant $\mathrm{scFv}-\mathrm{tP}$ protein-mediated siRNA delivery in clinics, due to the interaction of asFV with the HBsAg. To avoid such a disadvantage in siRNA-mediated HBV treatment, the present study presented a novel anti-HBV siRNA delivery system using recombinant preS1-tP proteins, in which the preS1 region of the HBV surface protein was applied to replace the previous fragment of antibodies against HBsAg.

Based on HepG2.2.15 cells stably expressing the human NTCP gene and the characterized HBV NLS siRNAs demonstrating inhibitory effects on the mRNA expression of HBV and production of two specific antigens in hepatocytes, the present study continued with the novel delivery method by 
expressing and purifying the recombinant preS1-tP proteins in E. coli. The in vitro co-incubation assay showed that the recombinant preS1-tP proteins efficiently entered into the HepG2.2.15 cells, and showed binding of DNA molecules with a high affinity. Finally, it was shown that the recombinant preS1-tP proteins successfully carried the HBV NLS siRNAs into the HepG2.2.15 cells stably expressing the human NTCP gene, which resulted in inhibition of the mRNA expression of $\mathrm{HBV}$, supported by significantly reduced HBsAg levels and cccDNA levels in the co-incubated HepG2.2.15 cells. Taken together, these experimental evidence demonstrated the potential of this recombinant preS1-tP protein-mediated siRNA delivery strategy in the clinical treatment of HBV and CHB.

Inspired by the rapid progress of siRNA-based anti-HBV therapies $(23,29)$, the establishment of this novel delivery system with improved efficiency may also be applied for the treatment of HBV with a focus on the siRNA-mediated inhibition of other associated genes in addition to HBV NLS. Beneficially, a single siRNA is capable of silencing multiple HBV transcripts responsible for synthesis of the core protein, surface protein and polymerase, due to the compact structure of the HBV genome and the overlapping of HBV transcripts (23). For example, Arrowhead ARC520, the first siRNA designed for $\mathrm{HBV}$ infection and $\mathrm{CHB}$ treatment to have entered clinical development, was shown to reduce the HBV DNA titer and suppress the expression of $\mathrm{HBsAg}, \mathrm{HBcAg}$ and $\mathrm{HBeAg}$ by targeting the HBV genome sequence upstream to the core promoter site $(30,31)$. However, the effect of ARC520 on inducing a reduction in $\mathrm{HBsAg}$ in $\mathrm{HBeAg}$-negative humans and animals was relatively weak, compared with the HBeAg-positive counterparts. For the development of more efficient and effective siRNAs for the treatment of $\mathrm{HBV}$, a number of novel siRNA programmes have been examined, including ARC-521, TKM-HBV, ALN-HBV and ISIS-HBV, and even locked nucleic acid (LNA) technology, which silences HBV transcripts using single-stranded oligodeoxyribonucleotides targeting mRNA molecules derived from HBV cccDNA (23). The novel siRNA-delivering system developed in the present study, using the recombinant preS1-tP proteins as carriers, may also be promising if applied for the treatment of HBV and CHB using these siRNAs, including ARC520 and LNA technology.

As a preliminary innovation in siRNA delivery, the technology demonstrated in the present study is far from clinical application. For example, the specificity of the recombinant preS1-tP proteins in delivering inhibitory siRNAs into HBV-positive liver cells requires carefully assessment using both in vitro and in vivo models prior to its further development for clinical experiments. The present study was limited as the efficiency of such a siRNA-delivering system has not been verified in an animal model. Further confirmation using $\mathrm{HBV}$ and $\mathrm{CHB}$ animal models may produce more convincing data and a basis for its application in the clinical treatment of patients with $\mathrm{CHB}$, with a focus required on the delivery specificity and safety.

In conclusion, the present study established HepG2.2.15 cells stably expressing the human NTCP gene as a cellular model for the development of a novel siRNA-delivering system for anti-HBV treatment. The recombinant preS1-tP proteins demonstrated capacity for carrying the HBV NLS siRNAs into the HepG2.2.15 cells, and subsequently resulting in the significant suppression of HBV replication, cccDNA formation and HBV-specific antigen expression. These results showed a promising and improved technique for the application of siRNA-based anti-CHB therapy, which may also inspire future innovation in the development of novel methods for $\mathrm{HBV}$ eradication and the treatment of $\mathrm{CHB}$.

\section{Acknowledgements}

Not applicable.

\section{Funding}

This study was supported by the National Natural Science Foundation of China (grant no. 81401706), the Chinese Foundation for Hepatitis Prevention and Control - Tianqing Liver Disease Research Fund Subject (grant no. TQGB20150138) and the Science Research Project of Henan Province (grant no. 142300410372 ).

\section{Availability of data and materials}

The analyzed data sets generated during the study are available from the corresponding author on reasonable request.

\section{Authors' contributions}

YK conceived and designed this project, and provided financial support. YZ performed the experiments, analyzed the data and drafted the manuscript. ZL and JS participated in study design, study implementation and manuscript revision. All authors read and approved the final manuscript.

\section{Ethics approval and consent to participate}

Not applicable.

\section{Consent for publication}

Not applicable.

\section{Competing interests}

The authors declare that they have no competing interests.

\section{References}

1. Carey WD: The prevalence and natural history of hepatitis B in the 21st century. Cleve Clin J Med 76 (Suppl 3): S2-S5, 2009.

2. Lai M and Liaw YF: Chronic hepatitis B: Past, present, and future. Clin Liver Dis 14: 531-546, 2010.

3. Lavanchy D: Hepatitis B virus epidemiology, disease burden, treatment, and current and emerging prevention and control measures. J Viral Hepat 11: 97-107, 2004.

4. Liu J, Zhang S, Wang Q, Shen H, Zhang M, Zhang Y, Yan D and Liu M: Seroepidemiology of hepatitis B virus infection in 2 million men aged 21-49 years in rural China: A population-based, cross-sectional study. Lancet Infect Dis 16: 80-86, 2015.

5. Lutgehetmann M, Volz T, Köpke A, Broja T, Tigges E, Lohse AW, Fuchs E, Murray JM, Petersen J and Dandri M: In vivo proliferation of hepadnavirus-infected hepatocytes induces loss of covalently closed circular DNA in mice. Hepatology 52: 16-24, 2010 . 
6. Spangenberg HC, Thimme R and Blum HE: Tracking cccDNA in chronic HBV infection. Hepatology 39: 1736-1738, 2004.

7. Levrero M, Testoni B and Zoulim F: HBV cure: Why, how, when? Curr Opin Virol 18: 135-143, 2016.

8. Daoud FS: Branchial cyst: An often forgotten diagnosis. Asian J Surg 28: 174-178, 2005.

9. Kann M, Bischof A and Gerlich WH: In vitro model for the nuclear transport of the hepadnavirus genome. J Virol 71: 1310-1316, 1997.

10. Li GQ, Gu HX, Li D and Xu WZ: Inhibition of Hepatitis B virus cccDNA replication by siRNA. Biochem Biophys Res Commun 355: 404-408, 2007.

11. Watashi K, Urban S, Li W and Wakita T: NTCP and Beyond: Opening the door to unveil hepatitis B virus entry. Int J Mol Sci 15: 2892-2905, 2014.

12. Abou-Jaoudé G and Sureau C: Entry of hepatitis delta virus requires the conserved cysteine residues of the hepatitis $\mathrm{B}$ virus envelope protein antigenic loop and is blocked by inhibitors of thiol-disulfide exchange. J Virol 81: 13057-13066, 2007.

13. Yan H, Zhong G, Xu G, He W, Jing Z, Gao Z, Huang Y, Qi Y, Peng B, Wang $\mathrm{H}$, et al: Sodium taurocholate cotransporting polypeptide is a functional receptor for human hepatitis B and D virus. Elife 1: e00049, 2012.

14. Claro da Silva T, Polli JE and Swaan PW: The solute carrier family 10 (SLC10): Beyond bile acid transport. Mol Aspects Med 34: 252-269, 2013.

15. Kullak-Ublick GA, Beuers U and Paumgartner G: Molecular and functional characterization of bile acid transport in human hepatoblastoma HepG2 cells. Hepatology 23: 1053-1060, 1996.

16. Kotani N, Maeda K, Debori Y, Camus S, Li R, Chesne C and Sugiyama Y: Expression and transport function of drug uptake transporters in differentiated HepaRG cells. Mol Pharm 9: 3434-3441, 2012

17. Wen WH, Liu JY, Qin WJ, Zhao J, Wang T, Jia LT, Meng YL, Gao H, Xue CF, Jin BQ, et al: Targeted inhibition of HBV gene expression by single-chain antibody mediated small interfering RNA delivery. Hepatology 46: 84-94, 2007.

18. Wen WH, Qin WJ, Gao H, Zhao J, Jia LT, Liao QH, Meng YL, Jin BQ, Yao LB, Chen SY, et al: An hepatitis B virus surface antigen specific single chain of variable fragment derived from a natural immune antigen binding fragment phage display library is specifically internalized by HepG2.2.15 cells. J Viral Hepat 14: 512-519, 2007

19. Manasseh D: Protamine binding location on DNA. 1995.

20. Ko C, Park WJ, Park S, Kim S, Windisch MP and Ryu WS: The FDA-approved drug irbesartan inhibits HBV-infection in HepG2 cells stably expressing sodium taurocholate co-transporting polypeptide. Antivir Ther 20: 835-842, 2015.
21. Mcfarland AP, Horner SM, Jarret A, Joslyn RC, Bindewald E, Shapiro BA, Delker DA, Hagedorn CH, Carrington M, Gale M Jr, etal:Thefavorable IFNL3 genotype escapesmRNAdecay mediated by AU-rich elements and hepatitis C virus-induced microRNAs. Nat Immunol 15: 72-79, 2014.

22. Urban TJ, Thompson AJ, Bradrick SS, Fellay J, Schuppan D, Cronin KD, Hong L, McKenzie A, Patel K, Shianna KV, et al: IL28B genotype is associated with differential expression of intrahepatic interferon-stimulated genes in patients with chronic hepatitis C. Hepatology 52: 1888-1896, 2010.

23. Gane EJ: Future anti-HBV strategies. Liver Int 37 (Suppl 1): S40-S44, 2017

24. Veloso Alves Pereira I, Buchmann B, Sandmann L, Sprinzl K, Schlaphoff V, Döhner K, Vondran F, Sarrazin C, Manns MP, Pinto Marques Souza de Oliveira C, et al: Primary biliary acids inhibit hepatitis D virus (HDV) entry into human hepatoma cells expressing the sodium-taurocholate cotransporting polypeptide (NTCP). PLoS One 10: e0117152, 2015.

25. Shen ZW, Luo MY, Hu HH, Zhou H, Jiang HD, Yu LS and Zeng S: Screening and verifying potential NTCP inhibitors from herbal medicinal ingredients using the LLC-PK1 cell model stably expressing human NTCP. Chin J Nat Med 14: 549-560, 2016.

26. Donkers J, Zehnder B, van Westen GJP, Kwakkenbos MJ, IJzerman AP, Oude Elferink RPJ, Beuers U, Urban S and van de Graaf SFJ: Reduced hepatitis B and D viral entry using clinically applied drugs as novel inhibitors of the bile acid transporter NTCP. Sci Rep 7: 15307, 2017.

27. Chen YM, Yin B, Guo XC, Wang W, Zheng Q, Wang F, Sun D, Li D, Ren G and Yin J: Selection of affinity-improved neutralizing human scFv against HBV PreS1 from CDR3 VH/VL mutant library. Biologicals 44: 271-275, 2016.

28. Wang H, Yang Y, Wang W, Guan B, Xun M, Zhang H, Wang ZL and Zhao Y: Construction and verification of anti-MM scFv-tP fusion protein expression vector. Nan Fang Yi Ke Da Xue Xue Bao 1149-1155, 2017 (In Chinese).

29. Marimani M, Hean J, Bloom K, Ely A and Arbuthnot P: Recent advances in developing nucleic acid-based HBV therapy. Future Microbiol 8: 1489-1504, 2013.

30. Xu Z, Chavez D, Guerra B, Littlejohn M, Peterson R, Locarnini S, Gish R, Anzalone C, Kanner S, Goetzmann J, et al: Treatment of chronically HBV-infected chimpanzees with RNA interference therapeutic ARC-520 LED to potent reduction of viral MRNA, DNA and proteins without observed drug resistance. J Hepatol 64: S398-S398, 2016.

31. Yuen MF, Chan HLY, Liu K, Given BD, Schluep T, Hamilton J, Lai CL, Locarnini SA, Lau JYN, Ferrari C and Gish RG: Differential reductions in viral antigens expressed from CCCDNAVS integrated DNA in treatment Naïve HBEAG positive and negative patients with chronic HBV after RNA interference therapy with ARC-520. J Hepatol 64: S390-S391, 2016. 